\title{
Clinical outcomes with olanzapine long-acting injection: impact of the 3-hour observation period on patient satisfaction and well-being
}

This article was published in the following Dove Press journal:

Neuropsychiatric Disease and Treatment

25 October 2016

Number of times this article has been viewed

Ernie Anand'

Lovisa Berggren ${ }^{2}$

John Landry ${ }^{3}$

Ágoston Tóth ${ }^{4}$

Holland C Detke ${ }^{5}$

'Neuroscience Medical Affairs, Eli Lilly \& Company Ltd, Windlesham, UK; ${ }^{2}$ Global Statistical Sciences, Lilly Deutschland $\mathrm{GmbH}$, Bad Homburg, Germany; ${ }^{3}$ Global Statistical Sciences, Eli Lilly Canada Inc., Toronto, ON, Canada: ${ }^{4}$ Neuroscience, Lilly Hungary, Budapest, Hungary; ${ }^{5}$ Psychiatry and Pain Disorders, Lilly Research Laboratories, Eli Lilly and Company, Indianapolis, IN, USA
Correspondence: Ernie Anand Neuroscience Medical Affairs, Eli Lilly \& Company Ltd, Lilly Research Center Erl Wood Manor, Sunninghill Road, Windlesham, Surrey, UK, GU 20 6PH Tel +44 I276 483063

Email anand_ernie@lilly.com
Background: The objective of the present analysis is to determine the impact of the 3-hour observation period for olanzapine long-acting injection (LAI) on patient satisfaction and wellbeing by comparing data collected before and after its implementation.

Methods: This is a post hoc analysis of patients treated with olanzapine LAI in 1) a 6-month fixed-dose randomized controlled trial and/or 2) a 6-year open-label safety study. This analysis was limited to patients with schizophrenia who were treated with olanzapine LAI consistent with the approved indication and dosing recommendations of the European Union Summary of Product Characteristics ( $\mathrm{N}=966)$. Of the 966 patients, the analysis further focused only on those patients who received both 1) at least one injection before the implementation of the 3-hour observation period and 2) at least one injection after implementation of the 3-hour observation period ( $\mathrm{N}=487$ ). Patient satisfaction was assessed with the three-item Patient Satisfaction with Medication Questionnaire-Modified. Responses were averaged across all postbaseline visits occurring before (ie, without) the implementation of the 3-hour observation period and across all postbaseline visits occurring after (ie, with) the implementation of the 3-hour observation period. In addition, the rate of postinjection delirium/sedation syndrome events was calculated.

Results: There was no meaningful change after implementation of the 3-hour observation period in satisfaction (before: mean $[\mathrm{SD}]=4.0[1.02]$ and after: mean $[\mathrm{SD}]=4.1[0.82]$ ), preference for olanzapine LAI over oral medication (before: mean $[\mathrm{SD}]=4.0[0.90]$ and after: mean $[\mathrm{SD}]=4.1$ [0.77]), or ratings of satisfaction regarding side effects (before: mean [SD] $=1.9$ [0.79] and after: mean $[\mathrm{SD}]=1.8[0.60])$. For the total population $(\mathrm{N}=966)$, postinjection delirium/sedation syndrome occurred in $26(0.07 \%)$ of 38,010 injections.

Conclusion: For patients with schizophrenia receiving treatment with olanzapine LAI, the 3-hour observation period had no impact on their satisfaction with the medication or on their subjective well-being.

Keywords: olanzapine long-acting injection, observation period, schizophrenia, Europe

\section{Introduction}

Olanzapine long-acting injection (LAI), a pamoate salt depot formulation of olanzapine, was formally introduced into clinical practice for the treatment of schizophrenia in 2008 following nearly a decade of prior research and development. ${ }^{1}$ In 2006 , during this research and development program, an important safety signal was detected, namely postinjection delirium/sedation syndrome (PDSS). PDSS is believed to be associated with vessel injury during the injection process, resulting in accidental intravascular entry of a portion of the dose. Because olanzapine LAI is a salt-based formulation, it is more soluble in blood than in muscle tissue, resulting in symptoms consistent 
with olanzapine intoxication, particularly excessive sedation (which could include coma) and/or delirium. ${ }^{2-4}$

The identification of PDSS ultimately resulted in the European label for olanzapine LAI having a risk minimization plan associated with it, the key elements being a 3-hour observation period and for patients to be accompanied home at the end of this 3-hour time period. ${ }^{3}$ In 2013, the European Medicines Agency announced that the safety signal of olanzapine LAI was sufficient to warrant the removal of the requirement of patients to be accompanied home, but the mandatory stipulation of the 3-hour observation period still remains. ${ }^{3,5}$

The objective of the present analysis was to determine the impact of the 3-hour observation period on patient satisfaction and well-being by comparing data collected before and after its implementation.

\section{Methods}

\section{Patients and design}

This is a post hoc analysis of data from two studies. Study F1D-MC-HGKA was a maintenance study in which patients (18-75 years of age) with schizophrenia were stabilized on oral olanzapine for 4-8 weeks and then randomized to 24 weeks of double-blind treatment with olanzapine LAI (150 mg every 2 weeks, $405 \mathrm{mg}$ every 4 weeks, $300 \mathrm{mg}$ every 2 weeks, or $45 \mathrm{mg}$ every 4 weeks [total $\mathrm{N}=743$ ]) or their stable oral olanzapine dose $(\mathrm{N}=322) .{ }^{6}$ Study F1D-MC-HGKB was a 6-year, single-arm, open-label Phase III study of olanzapine LAI in patients (18-76 years of age; $\mathrm{N}=931)$ with schizophrenia or schizoaffective disorder from three feeder studies (F1D-MC-HGJZ, F1D-MC-HGKA, and F1D-EW-LOBS). ${ }^{7}$ In that study, patients received $210 \mathrm{mg}$ olanzapine LAI at the first open-label visit. Patients received doses between $45 \mathrm{mg}$ and $405 \mathrm{mg}$ of olanzapine LAI at 2-week, 3-week, or 4-week intervals. Dosing after the first visit was determined on the basis of the investigators' clinical judgment.

The studies were conducted in accordance with ethics principles derived from international ethics guidelines, including the Declaration of Helsinki and the Council for International Organizations of Medical Sciences (CIOMS) International Ethical Guidelines, the International Conference on Harmonization (ICH) Good Clinical Practices (GCP) Guideline, and applicable laws and regulations. In addition, the study protocols were approved by ethical review boards at each site (Table $\mathrm{S} 1)$. After receiving a complete description of the study, all patients and/or their authorized legal representatives provided written informed consent before participation.
The present analysis was limited to patients who were treated with olanzapine LAI consistent with the approved indication and dosing recommendations of the European Union (EU) Summary of Product Characteristics (SmPC) ${ }^{5}$ that is, only those patients who met the diagnostic criteria for schizophrenia according to the Diagnostic and Statistical Manual of Mental Disorders, fourth edition, Text Revision, ${ }^{8}$ were included and patients with schizoaffective disorder were excluded. Patients who received oral olanzapine supplementation and who had a total daily dose of $>20 \mathrm{mg} / \mathrm{d}$ oral olanzapine equivalent were also excluded. This patient population ( $\mathrm{N}=966)$ was used in the calculation of the rate of PDSS events. For the analysis of patient satisfaction and well-being, the patient population was further refined to include only those patients who received both 1) at least one injection before the implementation of the 3-hour observation period and 2) at least one injection with the 3-hour observation period $(\mathrm{N}=487)$.

\section{Scales and measures}

All questionnaires and assessments (except the Clinical Global Impressions-Severity [CGI-S]) were completed every 6 months.

Patient satisfaction was assessed with the three-item Patient Satisfaction with Medication Questionnaire-Modified (PSMQ-Mod): satisfaction with current depot medication, preference between current depot medication and previous oral medications, and comparison of side effects between current depot medication and previous oral medications. The PSMQ-Mod uses a 5-point scale, with 5 representing highest satisfaction/preference for olanzapine LAI on the first two items and a score of 1 representing fewer side effects with olanzapine LAI on the third item. ${ }^{9}$

Patient well-being was assessed with the Subjective WellBeing Under Neuroleptics Scale-Short Form (SWNS). The SWNS has five subscores (self-control, emotional regulation, mental functioning, physical functioning, and social integration), each consisting of four questions with a total of 20 statements (ten positive and ten negative) and a possible total score of 20-120, with higher scores indicating greater subjective well-being. ${ }^{10}$

The 36-item Short Form Health Survey (SF-36) is a multipurpose, short-form patient-reported health survey used to evaluate overall physical and mental health. The SF-36 has eight subscales: bodily pain, general health, physical functioning, role-physical (contributing to the SF-12 physical score), mental health, role-emotional, social functioning, and vitality (contributing to the SF-12 mental score). Each 
scale has a total possible score of $0-100$, with higher scores indicating less disability. ${ }^{11}$

The Quality of Life (QoL) scale was used to measure the impact of schizophrenia symptoms on occupational, social, and psychological functioning in patients with schizophrenia. The total score of the scale is the sum of 21 items, with each item being rated on a 7-point scale. Four categories contained in the scale are intrapsychic foundations (items 13-17 and 20 and 21), interpersonal relations (items 1-8), instrumental role (items 9-12), and common objects and activities (items 18 and 19), with higher scores indicating less impairment. ${ }^{12}$

The Positive and Negative Syndrome Scale (PANSS) was used to evaluate the symptoms of schizophrenia. The scale has a total possible score of 30-210, with higher scores indicating more severe symptomatology. ${ }^{13}$

The CGI-S was used to assess the severity of schizophrenia. The single question is rated on a 7-point scale ranging from 1 (normal, not at all ill) to 7 (among the most extremely ill). ${ }^{14}$ The CGI-S was assessed at quarterly visits.

The rate of PDSS events was also summarized.

\section{Statistical analyses}

All patients who received both at least one injection before the implementation of the 3-hour observation period and at least one injection with the 3 -hour observation period were included in this post hoc analysis. Demographics and baseline characteristics were summarized by their frequencies. For all health outcome measures and efficacy measures, responses were averaged within patients across all postbaseline visits occurring before (ie, without) the implementation of the 3-hour observation period and across all postbaseline visits occurring after (ie, with) the implementation of the 3-hour observation period. No adjustments were made to variance estimates to account for the different number of measurements taken between patients. Due to exploratory nature of the analyses, only descriptive statistics are provided.

\section{Clinical trial registration}

The trials from which data are reported here were registered on ClinicalTrials.gov as NCT00088465 and NCT00088491.

\section{Results}

A total of 966 patients were treated with olanzapine LAI consistent with the approved indication and dosing recommendations of the EU Summary of Product Characteristics (SmPC). This patient population was used for the calculation of the rate of PDSS events. Of these 966 patients, the present analysis further focused on those patients $(n=487)$ who received at least one injection before the implementation of the 3-hour observation period and at least one injection with the 3-hour observation period. The mean (SD) age of these patients was 38.2 (11.75) years, with most patients being male (66.3\%) and Caucasian (67.1\%) (Table 1).

Among patients who were treated both before and after implementation of the 3-hour observation period, there was no meaningful change in their satisfaction with olanzapine LAI (before: mean $[\mathrm{SD}]=4.0[1.02]$ and after: mean $[\mathrm{SD}]=4.1$ $[0.82])$, preference for olanzapine LAI over oral medication (before: mean $[\mathrm{SD}]=4.0[0.90]$ and after: mean $[\mathrm{SD}]=4.1$ [0.77]), or ratings of satisfaction regarding side effects (before: mean $[\mathrm{SD}]=1.9[0.79]$ and after: mean $[\mathrm{SD}]=1.8$ [0.60]) (Figure 1).

Total scores on the SWNS were similar before and after implementation of the 3-hour observation period (before: mean $[\mathrm{SD}]=87.8$ [14.28] and after: mean $[\mathrm{SD}]=88.6$ [13.14]). Similar results were reported for subscale scores of self-control, emotional regulation, mental functioning, physical functioning, and social integration (Figure 2).

No meaningful changes were reported on any of the subscales of the SF-36, including the SF-12 physical subscale (before: mean $[\mathrm{SD}]=50.8[6.68]$ and after: mean $[\mathrm{SD}]=50.8$ [6.69]) and SF-12 mental subscale (before: mean $[\mathrm{SD}]=45.2$ [9.49] and after: mean [SD] $=46.6[8.35]$ ), scales after implementation of 3-hour observation period compared to before implementation (Figure 3).

On the QoL scale, the total score was numerically higher after implementation of 3-hour observation period compared to before implementation of 3-hour observation period, but the difference in scores was not considered clinically meaningful (total score, before: mean [SD] $=70.5$ [19.7] and after: mean $[\mathrm{SD}]=77.3[21.07])$. Similar results were reported in the subscale scores of the QoL (Figure 4).

Table I Demographics

\begin{tabular}{ll}
\hline Characteristic & Patients (N=487) \\
\hline Age, mean (SD) & $38.2($ II.75) \\
Sex, $\mathrm{n}(\%)$ & \\
$\quad$ Female & $164(33.7)$ \\
$\quad$ Male & $323(66.3)$ \\
Race, $\mathrm{n}(\%)$ & \\
African & $30(6.2)$ \\
Caucasian & $327(67.1)$ \\
East Asian & $22(4.5)$ \\
Hispanic & $92(18.9)$ \\
Native American & $\mathrm{I}(0.2)$ \\
West Asian (Indian subcontinent) & $\mathrm{I}(3.1)$ \\
\hline
\end{tabular}

Notes: N, number of patients; $n$, number of affected patients. 


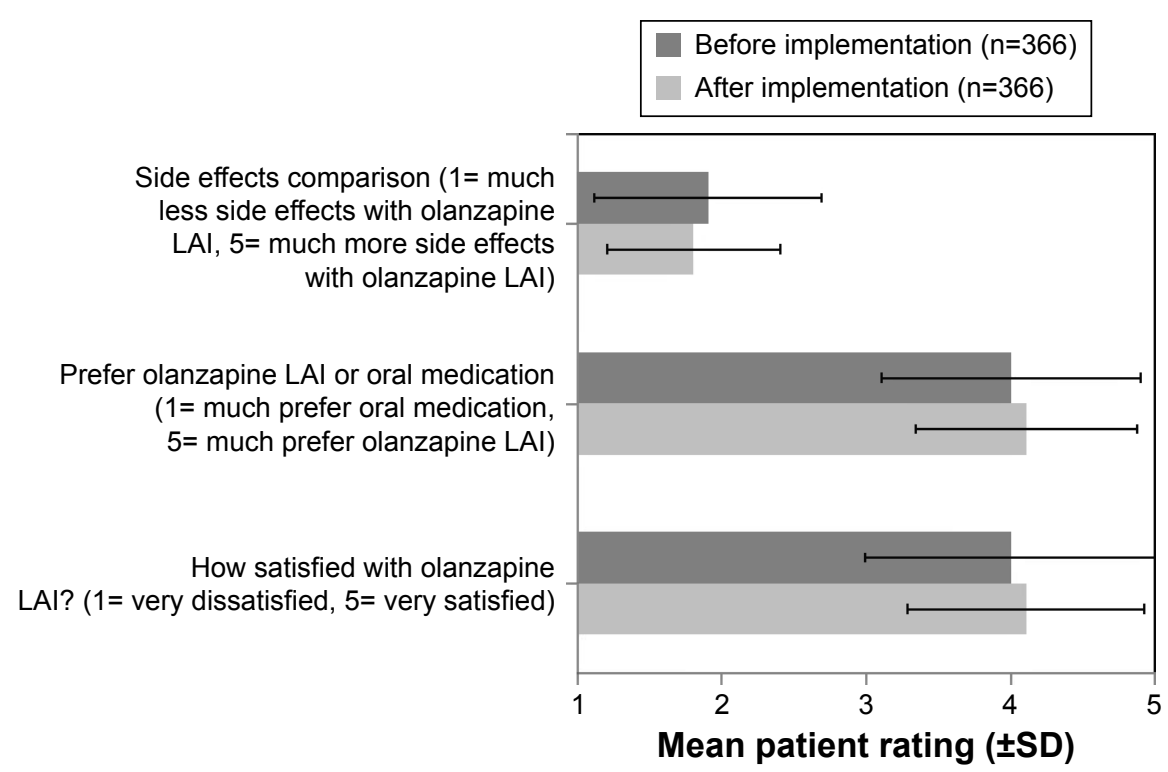

Figure I Patient responses on the PSMQ-Mod: before and after implementation of the 3-hour observation period.

Note: $n$, number of patients.

Abbreviations: LAI, long-acting injection; PSMQ-Mod, Patient Satisfaction with Medication Questionnaire-Modified.

The PANSS total score, as well as the positive symptoms, negative symptoms, and general psychopathology subscale scores, was similar before and after implementation of the 3-hour observation period (Figure 5). Scores on the CGI-S were also similar before and after implementation of the 3-hour observation period (before: mean [SD] $=2.7$ [0.91] and after: mean $[\mathrm{SD}]=2.5[0.86])$.

Of the 38,010 injections given to patients with schizophrenia who were treated with olanzapine LAI consistent with the approved indication and dosing recommendations of the EU SmPC (N=966), 26 (0.07\%) were followed by a PDSS event. The percentage of injections resulting in
PDSS events was similar before $(10 / 13,660=0.07 \%)$ and after $(16 / 24,350=0.07 \%)$ implementation of the 3 -hour observation period.

\section{Discussion}

On measures of patient satisfaction, well-being, health, QoL, and symptoms of schizophrenia, we found no evidence of an effect of the 3-hour observation period. On each measure, scores were only slightly different before and after implementation of the 3-hour observation period. Also, as expected, the percentage of injections resulting in a PDSS event was essentially the same before and after implementation of

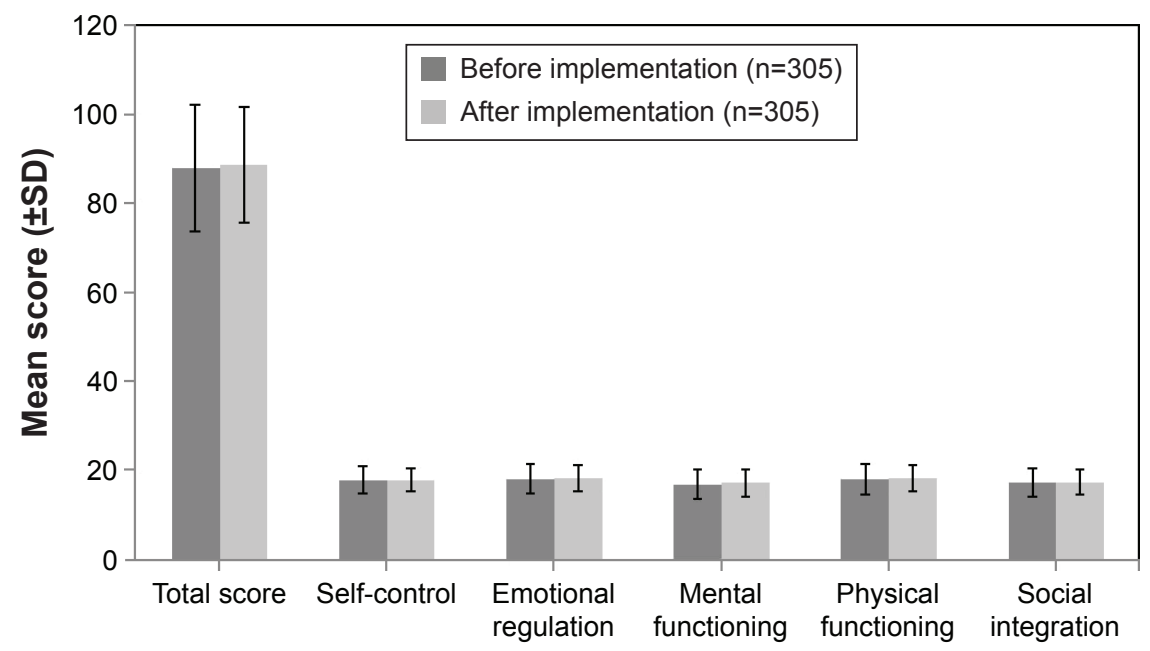

Figure 2 Patient responses on the SWNS: before and after implementation of the 3-hour observation period.

Note: $n$, number of patients.

Abbreviation: SWNS, subjective well-being under neuroleptics scale-short form. 


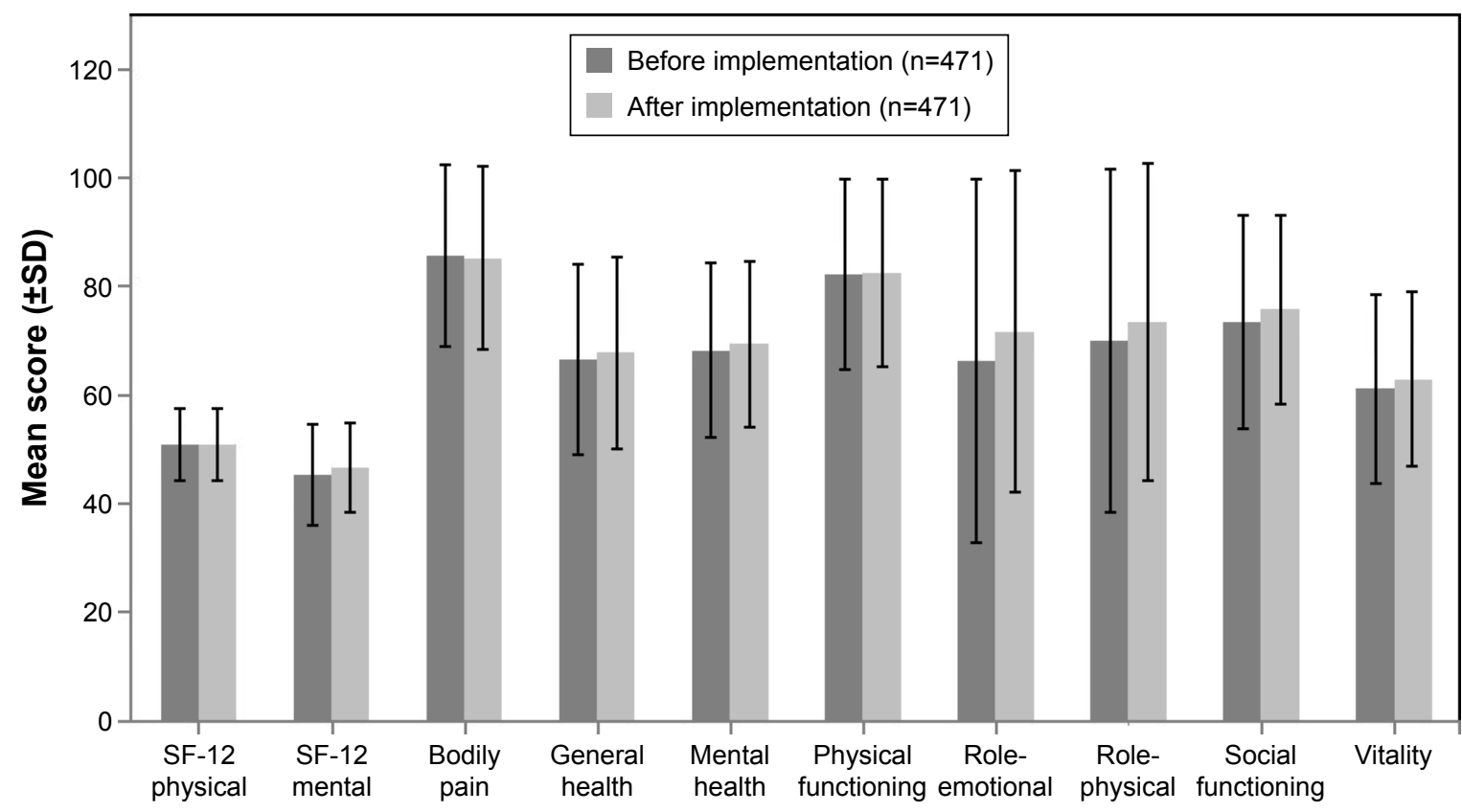

Figure 3 Patient responses on the SF-36: before and after implementation of the 3-hour observation period.

Notes: $\mathrm{n}=470$ (before and after implementation) for the SF- 12 physical, SF- 12 mental, and general health subscales. $n$, number of patients.

Abbreviations: SF-12, I2-Item Short Form Health Survey; SF-36, 36-Item Short Form Health Survey.

the 3-hour observation period. The percentage of PDSS events that occurred following injections with olanzapine LAI in this patient sample is similar to that previously reported $(<0.1 \%) .^{5}$

It should also be noted that, overall, these patients had relatively low symptom levels, as patients were generally clinically stable when they began treatment with olanzapine LAI in this clinical study. This is evident by their responses on the PANSS and CGI-S and supported by their responses on the SWNS, SF-36, and QoL scale.

Overall, on the PSMQ-Mod, the mean responses both before and after implementation indicated that patients experienced less side effects with olanzapine LAI and preferred olanzapine LAI compared to their oral medication and that they were satisfied with olanzapine LAI. As expected, these results agree with a previous report

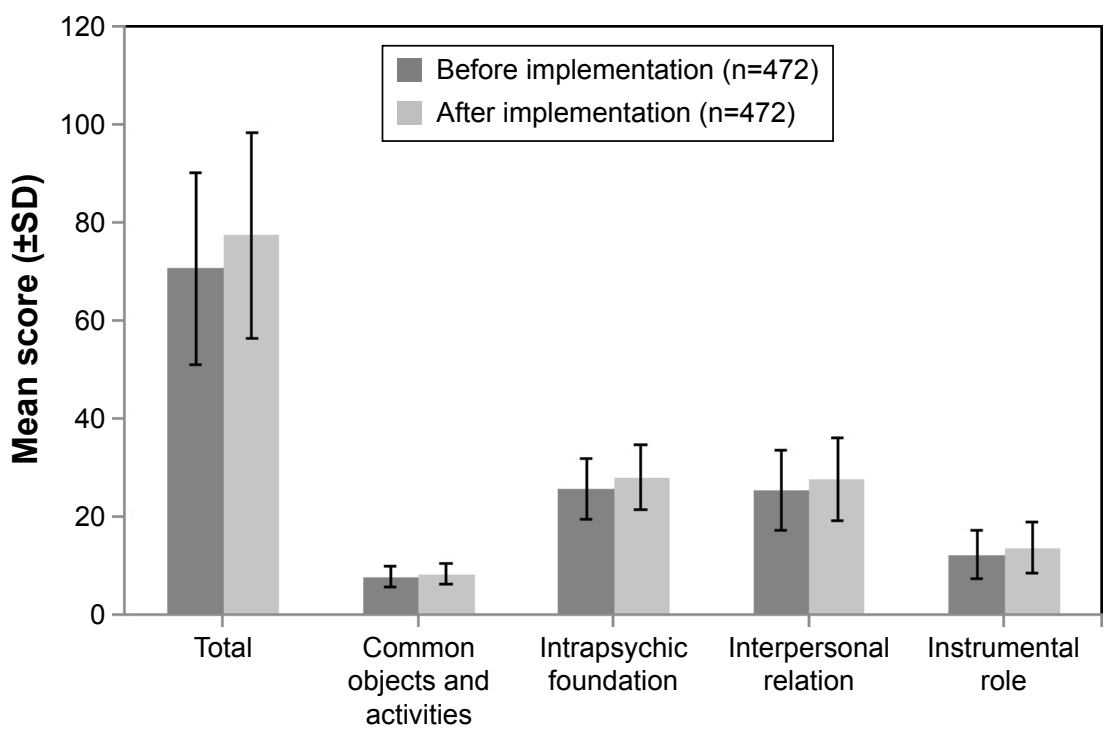

Figure 4 Patient responses on the QoL scale: before and after implementation of the 3-hour observation period.

Notes: $n=47$ I (before and after implementation) for the total score and the instrumental role subscale score. $n$, number of patients.

Abbreviation: QoL, quality of life. 


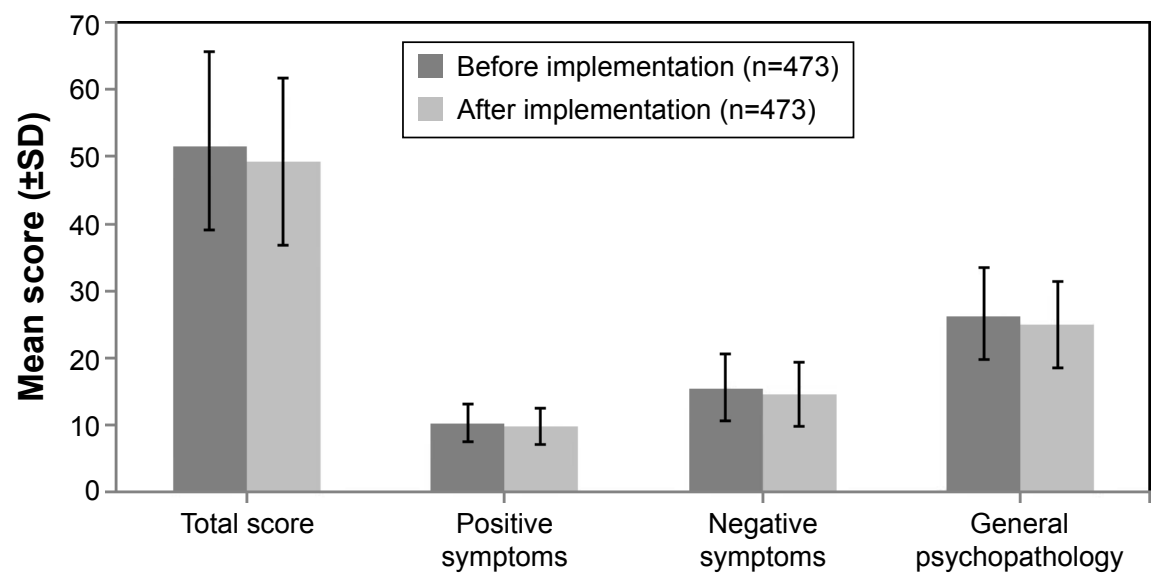

Figure 5 PANSS total and subscale scores before and after implementation of the 3-hour observation period.

Note: $n$, number of patients.

Abbreviation: PANSS, Positive and Negative Syndrome Scale.

(from a largely overlapping patient sample), indicating that $73.7 \%$ of patients being treated with olanzapine LAI were somewhat or very satisfied with their medication, 69.5\% preferred or much preferred olanzapine LAI versus previous oral medications, and $74.2 \%$ thought that olanzapine LAI was associated with "less" or "much less side effects". ${ }^{15}$

Though it might not be expected that measures of efficacy and well-being would be affected by the 3-hour observation period, it seems reasonable to hypothesize that the initiation of a 3-hour observation period would result in a decrease in satisfaction among olanzapine LAI patients because of the potential resulting inconvenience and interruption of daily routine. However, if this effect is influencing scores at all, it is counterbalanced by other factors. For example, one might expect scores to improve somewhat during the course of an open-label study as some patients continue to improve with treatment and others do not do as well and drop out. However, there is a less obvious possibility. Anecdotal evidence suggests that patients may find that additional time with their mental health care team may be a positive experience for them. Naber ${ }^{16}$ suggests that positive benefits (eg, patient acceptance and better therapeutic relationship) may result from regular contact with their health care providers. Indeed, the 3-hour observation period could well be further utilized by offering psychotherapy or psychoeducation. ${ }^{17}$

\section{Limitations}

There are a few limitations of this analysis that should be recognized. First, with no a priori sample size calculation, it is unclear whether the sample of subjects used was large enough to detect a difference in our measures, if one existed. Second, our conclusions are based on interpretation of a body of descriptive statistics rather than predefined inferential tests. Third, this analysis was limited to patients with schizophrenia who were treated with olanzapine LAI consistent with the approved indication and dosing recommendations of the EU SmPC. Results that included responses of patients with schizoaffective disorder, or patients who were given supplementary oral doses of olanzapine beyond those recommended by the EU SmPC, could have been different.

\section{Conclusion}

For patients with schizophrenia receiving treatment with olanzapine LAI consistent with the approved indication and dosing recommendations of the EU SmPC, the 3-hour observation period had no impact on their satisfaction with the medication and on their subjective well-being, overall health, QoL, or symptoms of schizophrenia. It is hoped that these results will help EU clinicians further define and refine how best they would like to use olanzapine LAI in treating their patients with schizophrenia, especially in keeping within the current requirements of the EU SmPC.

\section{Acknowledgments}

We would like to dedicate this publication to the memory of our coauthor and colleague, Doctor Marlene Popescu. Assistance with drafting/editing of the article was provided by inVentiv Health Clinical, funded by Eli Lilly and Company. The abstract of this paper was presented at the 28th ECNP Congress, August 29 to September 1, 2015, Amsterdam, the Netherlands, as a poster presentation with interim findings. The poster's abstract was published in "Poster Abstracts" in European Neuropsychopharmacology September 2015, volume 25, supplement 2, pages S535-S536 
(P.3.d.094). This research was funded by Eli Lilly and Company, Indianapolis, IN, USA.

\section{Disclosure}

All authors are the employees and minor shareholders of Eli Lilly and Company. The authors report no other conflicts of interest in this work.

\section{References}

1. Frampton JE. Olanzapine long-acting injection: a review of its use in the treatment of schizophrenia. Drugs. 2010;70(17):2289-2313.

2. Detke HC, McDonnell DP, Brunner E, et al. Post-injection delirium/ sedation syndrome in patients with schizophrenia treated with olanzapine long-acting injection, I: analysis of cases. BMC Psychiatry. 2010;10:43.

3. Luedecke D, Schöttle D, Karow A, Lambert M, Naber D. Post-injection delirium/sedation syndrome in patients treated with olanzapine pamoate: mechanism, incidence, and management. CNS Drugs. 2015; 29(1):41-46.

4. McDonnell DP, Detke HC, Bergstrom RF, et al. Post-injection delirium/ sedation syndrome in patients with schizophrenia treated with olanzapine long-acting injection, II: investigations of mechanism. BMC Psychiatry. 2010;10:45.

5. ZypAdhera ${ }^{\circledR}$ (olanzapine) [Summary of Product Characteristics]. Indianapolis, IN: Eli Lilly and Company; 2015.

6. Kane JM, Detke HC, Naber D, et al. Olanzapine long-acting injection: a 24-week, randomized, double-blind trial of maintenance treatment in patients with schizophrenia. Am J Psychiatry. 2010;167(2):181-189.

7. McDonnell DP, Landry J, Detke HC. Long-term safety and efficacy of olanzapine long-acting injection in patients with schizophrenia or schizoaffective disorder: a 6-year, multinational, single-arm, open-label study. Int Clin Psychopharmacol. 2014;29(6):322-331.
8. American Psychiatric Association. Diagnostic and Statistical Manual of Mental Disorders. 4th ed. Text Revision ed. Washington, DC: American Psychiatric Association; 2000.

9. Kalali A. Patient satisfaction with, and acceptability of, atypical antipsychotics. Curr Med Res Opin. 1999;15(2):135-137.

10. Naber D, Moritz S, Lambert M, et al. Improvement of schizophrenic patients' subjective well-being under atypical antipsychotic drugs. Schizophr Res. 2001;50(1-2):79-88.

11. Ware JE, Snow KK, Kosinski M, Gandek B. SF-36 Health Survey Manual and Interpretation Guide. Boston: The Health Institute, New England Medical Center; 1993.

12. Heinrichs DW, Hanlon TE, Carpenter WT Jr. The Quality of Life Scale: an instrument for rating the schizophrenic deficit syndrome. Schizophr Bull. 1984;10(3):388-398.

13. Kay SR, Fiszbein A, Opler LA. The Positive and Negative Syndrome Scale (PANSS) for schizophrenia. Schizophr Bull. 1987;13(2): 261-276.

14. Guy W. ECDEU Assessment Manual for Psychopharmacology. Rockville, MD: U.S. Dept. of Health, Education, and Welfare, Public Health Service, Alcohol, Drug Abuse, and Mental Health Administration, National Institute of Mental Health, Psychopharmacology Research Branch, Division of Extramural Research Programs; 1976: 217-222.

15. Anand E, Berggren L, Deix C, Tóth Á, McDonnell DP. A 6-year open-label study of the efficacy and safety of olanzapine long-acting injection in patients with schizophrenia: a post hoc analysis based on the European label recommendation. Neuropsychiatr Dis Treat. 2015; 11:1349-1357.

16. Naber D. Olanzapine pamoate for the treatment of schizophrenia. Expert Opin Pharmacother. 2011;12(4):627-633.

17. Schöttle D, Kuhnigk O, Naber D. Drug safety evaluation of olanzapine pamoate. Expert Opin Drug Saf. 2013;12(6):897-903.
Neuropsychiatric Disease and Treatment

\section{Publish your work in this journal}

Neuropsychiatric Disease and Treatment is an international, peerreviewed journal of clinical therapeutics and pharmacology focusing on concise rapid reporting of clinical or pre-clinical studies on a range of neuropsychiatric and neurological disorders. This journal is indexed on PubMed Central, the 'PsycINFO' database and CAS,

\section{Dovepress}

and is the official journal of The International Neuropsychiatric Association (INA). The manuscript management system is completely online and includes a very quick and fair peer-review system, which is all easy to use. Visit http://www.dovepress.com/testimonials.php to read real quotes from published authors. 